\title{
SUFFICIENT CONDITIONS FOR MATCHINGS
}

\author{
by IAN ANDERSON \\ (Received 7th October 1971)
}

\section{Introduction}

A graph $G$ is said to possess a perfect matching if there is a subgraph of $G$ consisting of disjoint edges which together cover all the vertices of $G$. Clearly $G$ must then have an even number of vertices. A necessary and sufficient condition for $G$ to possess a perfect matching was obtained by Tutte (3). If $S$ is any set of vertices of $G$, let $p(S)$ denote the number of components of the graph $G-S$ with an odd number of vertices. Then the condition

$$
\text { for all } S, p(S) \leqq|S|
$$

is both necessary and sufficient for the existence of a perfect matching. A simple proof of this result is given in (1).

We consider certain conditions which are sufficient although not necessary. Roughly speaking, $G$ will have a perfect matching if there are enough edges. For example, if $|V(G)|=n, n$ even, where $V(G)$ denotes the set of vertices of $G$, and if each vertex is of degree $\geqq \frac{1}{2} n$, i.e. if each vertex has at least $\frac{1}{2} n$ edges incident with it, then it is almost trivial (see $\S 3$ ) to show that $G$ has a perfect matching. Instead of looking at each vertex separately, we can put a condition on the vertices collectively. If $X$ denotes any subset of $V(G)$, let

$$
\Gamma(X)=\{y \in V(G): y \text { is joined by an edge to at least one vertex in } X\} .
$$

Following Woodall (4), we define

$$
\operatorname{melt}(G)=\max \{c: \forall X \subset V(G),|\Gamma(X)| \geqq \min (c|X|,|V(G)|)\} .
$$

Thus melt $(G)$ is the largest number $c$ such that any $k$ vertices of $G$ are collectively adjacent to at least $\min (c k, n)$ vertices. We have already (1) shown that, if $n$ is even,

$$
\text { melt }(G) \geqq \frac{4}{3} \Rightarrow G \text { has a perfect matching. }
$$

We note that this condition implies that each vertex is of degree $\geqq \frac{1}{4} n$. Indeed, we have in general

Lemma. If melt $(G) \geqq c>1$, then each vertex of $G$ has degree $\geqq \frac{c-1}{c} n$ where $n=|V(G)|$.

Proof. Suppose there is a vertex $v$ of degree $\leqq \frac{c-1}{c} n$. Then there are 
$\geqq \frac{n}{c}$ vertices none of which is joined by an edge to $v$. But these vertices must be joined to at least $c \cdot \frac{n}{c}=n$ vertices, a contradiction.

In the next section, we combine the two types of condition above to prove

Theorem 1. Let $G$ have $n$ vertices, $n$ even. Let $c$ be any fixed number, $\frac{1}{4} \leqq c \leqq \frac{1}{2}$, and suppose that

(i) each vertex is of degree $\geqq c n$,

(ii) melt $(G) \geqq \frac{3-4 c}{2-2 c}$.

Then $G$ possesses a perfect matching.

Note 1. $c=\frac{1}{2}$ gives the trivial result mentioned above, and $c=\frac{1}{4}$ gives the result (1).

Note 2. The theorem is also true for other values of $c$, but if $c>\frac{1}{2}$ condition (i) by itself is sufficient, whereas if $c<\frac{1}{4}$ then condition (ii) by itself suffices.

Note 3. Condition (ii) implies, by the lemma, that each vertex has degree $\geqq \frac{1-2 c}{3-4 c} n$, but this is less than $c n$ if $c>\frac{1}{4}$.

Note 4. The result is best possible. If $A, B$ are graphs let $A+B$ denote the graph obtained by joining every vertex of $A$ to every vertex of $B$. Take $A=a K_{3} \cup b K_{1}$ and $B=(a+b-2) K_{1}$ where $K_{n}$ denotes the complete graph on $n$ vertices.

Following the suggestion of the referee, who is to be thanked for his careful consideration of the original version of this paper, we shall deduce Theorem 1 from the following stronger theorem which is proved along the same lines but more simply.

Theorem 2. Let $G$ have $n$ vertices, $n$ even, and suppose that

$$
|\Gamma(X)| \geqq \min \left(2|X|-\frac{n}{2}, n\right)
$$

for all sets $X$ of vertices of $G$. Then either $G$ has a perfect matching or there exist subsets $X, Y$ of $V(G), X \nsubseteq Y$, such that

$|X|=\frac{1}{4}(3 n-6),|Y|=\frac{1}{4}(3 n-2),|\Gamma(X)|=2|X|-\frac{n}{2},|\Gamma(Y)|=2|Y|-\frac{n}{2}$.

An example of a graph in which the second possibility occurs is $G=3 K_{3}+K_{1}$. Theorem 2 is proved in the next section, but we now show that 
Theorem 2 implies Theorem 1. We assume Theorem 2 and the hypotheses of Theorem 1. Let $W$ be any set of vertices of $G$. If $|W|>(1-c) n$, then, since the degree of each vertex of $G$ is $\geqq c n$, we cannot have a vertex of $G$ joined to no vertex of $W$. Thus $|\Gamma(W)|=V(G)$. So suppose $|W| \leqq(1-c) n$. Then

$$
|\Gamma(W)| \geqq \frac{3-4 c}{2-2 c}|W| \geqq 2|W|-\frac{n}{2} .
$$

It follows from Theorem 2 that $G$ possesses a perfect matching unless there exist two sets $X, Y$ as in Theorem 2 . Then, by (2),

$$
\frac{3-4 c}{2-2 c}|W|=2|W|-\frac{n}{2}
$$

for $W=X$ and for $W=Y$, giving $|X|=|Y|$, a contradiction.

Theorem 2 is proved in the next section. In the remainder of this paper we shall generalize in one theorem both Theorem 1 and a result of Woodall (4) concerned with the maximum number of disjoint edges in a graph with no perfect matching. Woodall's argument was based on that of (1), and now we in turn extend his result.

\section{Proof of Theorem 2}

We suppose there is no perfect matching of $G$. Then by Tutte's theorem there is a set $S$ of vertices of $G$ for which $p(S)>|S|$. Using the fact that $p(S) \equiv|S|(\bmod 2)$, we must then have

$$
p(S) \geqq|S|+2 .
$$

Case 1. Suppose that $|S| \geqq \frac{1}{4}(n-6)$. Let $m$ denote the number of 1components of $G-S$ (i.e. the number of components with just one vertex). Then

whence

$$
\begin{aligned}
n & \geqq|S|+m+3(p(S)-m) \\
& \geqq 4|S|+6-2 m,
\end{aligned}
$$

But, if $m>0$,

$$
n-m \leqq \frac{3}{2} n-2|S|-3 \text {. }
$$

$$
\begin{aligned}
& n-m \geqq|\Gamma(G-S)| \geqq 2|G-S|-\frac{n}{2} \\
&=2 n-2|S|-\frac{n}{2},
\end{aligned}
$$

whence

$$
n-m \geqq \frac{3}{2} n-2|S|
$$


Since (4) and (5) contradict one another, we must have $m=0$. Thus, from (3),

i.e.

$$
n \geqq 4|S|+6
$$

whence

$$
|S| \leqq \frac{1}{4}(n-6)
$$

$$
|S|=\frac{1}{4}(n-6) \text {. }
$$

Equality here implies that each component of $G-S$ must have exactly 3 vertices. If we let $X$ denote the set of vertices in all but one of these components we then have $|X|=\frac{1}{4}(3 n-6)$ and $|\Gamma(X)| \leqq|X|+|S|=n-3=2|X|-\frac{n}{2}$. Similarly, if $Y$ denotes the same set with one more vertex of $G-S$ added, then we also have $|Y|=\frac{1}{4}(3 n-2)$ and $|\Gamma(Y)| \leqq|Y|+|S|=n-1=2|Y|-\frac{n}{2}$.

Case 2. Suppose now that $|S|<\frac{1}{4}(n-6)$. Let $h$ denote the number of vertices in all but the smallest component of $G-S$. Since there are $\geqq|S|+2$ components of $G-S$, containing between them $n-|S|$ vertices, we must have

$$
h \geqq \frac{|S|+1}{|S|+2}(n-|S|) \text {. }
$$

These $h$ vertices can be adjacent to at most $h+|S|<n$ vertices; on the other hand, they are by hypothesis joined to at least $2 h-\frac{n}{2}$ vertices. Thus

$$
h \leqq|S|+\frac{n}{2}
$$

From (6) and (7), eliminating $h$, we obtain

a contradiction.

$$
|S| \geqq \frac{1}{4}(n-6)
$$

\section{Extension to imperfect matchings}

A related question is the following. Given a condition on a graph $G$ which does not imply that $G$ possesses a perfect matching, can we estimate how many disjoint edges can be found in $G$ ? Corresponding to the two types of condition already studied, we have the following results for a graph with $n$ vertices.

1. If each vertex is of degree $\geqq c n, 0 \leqq c \leqq \frac{1}{2}$, then we can find at least $[c n]$ disjoint edges.

2. If melt $(G) \geqq c$, then there are at least

$$
\begin{gathered}
\frac{c}{c+1} n \text { disjoint edges if } 0<c \leqq \frac{1}{2} \\
{\left[\frac{3 c-2}{3 c} n\right] \text { disjoint edges if } 1<c \leqq \frac{4}{3} .}
\end{gathered}
$$


Result 2 is due to Woodall (4), with (1) as the special case $c=\frac{4}{3}$. Result 1 is almost trivial (although best possible-consider a bipartite graph). For suppose that each vertex is of degree $\geqq k$, and that $h<k$ disjoint edges have so far been found. If no two remaining vertices are joined by an edge, select any two of them, say $v_{1}$ and $v_{2}$. Then it is easy to see that there must be a pair $v_{3}, v_{4}$ of vertices, joined by one of the edges already chosen, such that $v_{1}$ is joined to $v_{3}$ and $v_{2}$ to $v_{4}$. With this new pairing we now have $h+1$ disjoint edges, and the process can be repeated if $h+1<k$. We now state

Theorem 3. Let $G$ be a graph with $n$ vertices. Suppose that

(i) each vertex is of degree $\geqq d n$,

(ii) melt $(G) \geqq \frac{3-4 d-3 f}{2-2 d}$,

where $4 d+3 f \geqq 1,2 d+3 f \leqq 1, d \geqq 0, f \geqq 0$. Then $G$ possesses at least $\left[\frac{n}{2}(1-f)\right]$ disjoint edges.

The special case $f=0$ is Theorem 1 , and the case $f=\frac{1}{3}(1-4 d)$ is Woodall's result (9). The referee has suggested that it may be possible to deduce this result from an analogue to Theorem 2 in the same way as Theorem 1 was deduced from Theorem 2. However, we preserve here our original proof. Instead of Tutte's condition we use Berge's extension ((2); see also (4) for a simpler proof): for $G$ to possess at least $t$ disjoint edges, it is necessary and sufficient that $p(S)-|S| \leqq n-2 t$ for all sets $S$ of vertices of $G$. We shall in fact prove that, for all $S$,

$$
p(S) \leqq|S|+n f+\frac{5}{3}
$$

since this will imply that there are at least $\frac{n}{2}(1-f)-\frac{5}{6}$ and hence at least $\left[\frac{n}{2}(1-f)\right]$ disjoint edges.

\section{Proof of Theorem 3}

In view of the above remarks, we may suppose that there exists a set $S$ of vertices of $G$ such that

$$
p(S)>|S|+n f+\frac{5}{3}
$$

and show that this leads to a contradiction.

Case 1. $|S| \geqq d n$. Let $m$ denote the number of 1-components in $G-S$. If $m=0$,

$$
n \geqq|S|+3 p(S)>4|S|+3 f n \geqq(4 d+3 f) n \geqq n,
$$

so we must have $m>0$. Thus

$$
n-m \geqq|\Gamma(G-S)| \geqq \frac{3-4 d-3 f}{2-2 d}(n-|S|),
$$


whence

$$
m \leqq \frac{3-4 d-3 f}{2-2 d}|S|-\frac{1-2 d-3 f}{2-2 d} n .
$$

But we also have, from (3), ignoring the term $\frac{5}{3}$ in (10),

i.e.

$$
\begin{aligned}
& n>4|S|-2 m+3 n f, \\
& m>2|S|-\frac{1}{2}(1-3 f) n .
\end{aligned}
$$

Eliminating $m$ from (11) and (12), we obtain $|S|<d n$, a contradiction.

Case 2. $|S|<d n$. Here there can be no 1-components, so that each odd component contains at least

$$
\max (3, d n-|S|+1)
$$

vertices. From now on we can assume that $4 d+3 f>1$.

Case 2(a). Suppose there is at least one 3-component. Then (13) yields

Then (3) and (10) give

$$
d n-|S|=\beta, \quad 0<\beta \leqq 2 .
$$

so that

$$
n>4|S|+3 n f+5=(4 d+3 f) n-4 \beta+5
$$

$$
n(4 d+3 f-1)<4 \beta-5 \text {. }
$$

Considering on the other hand all but one of the odd components we have, from the definition of melt $(G)$,

$$
n-3 \geqq \frac{3-4 d-3 f}{2-2 d}(n-|S|-3) .
$$

Substituting for $|S|$ from (14), this gives

$$
n(1-d)(4 d+3 f-1) \geqq(3-\beta)(4 d+3 f-1)-6 d+2 \beta>2 \beta-6 d .
$$

Thus, by (15), we must have

whence

$$
2 \beta-6 d<(1-d)(4 \beta-5)
$$

It follows that

$$
d>\frac{1}{3}
$$

$$
4 d+3 f-1>\frac{1}{3} \text {. }
$$

(15) and (16), with $\beta \leqq 2$, now yield $n<9$, and a contradiction easily follows.

Case 2 (b). Suppose now there is no 3-component. Here we shall show that $|S|$ is bounded. First of all, if $|S|<\frac{1}{2} d n$, then

$$
n>|S|+\left(|S|+n f+\frac{5}{3}\right)\left(\frac{1}{2} d n+1\right)
$$

so that

$$
\frac{1}{2} d n|S|<|S|\left(2+\frac{1}{2} d n\right)<n\left(1-f-\frac{5}{6} d-\frac{1}{2} f d n\right) .
$$




\section{SUFFICIENT CONDITIONS FOR MATCHINGS}

If $d n<4$ then $|S|<1$ whereas, if $d n \geqq 4$, then $\frac{1}{2} f d n \geqq 2 f$ and (17) yields $|S|<6$. Secondly, if $\frac{1}{2} d n \leqq|S|<d n$, then

whence

$$
n>|S|+(|S|+n f)(d n-|S|+1)
$$

Thus

$$
d n-|S|<\frac{n+n f}{|S|+n f}-2<\frac{1+f}{\frac{1}{2} d+f}-2<8 \text {. }
$$

so that $n<80$. But

$$
\begin{aligned}
& 8+\frac{1}{6} n(1-5 f)>8+|S|>d n, \\
& 40>(6 d+5 f-1) n>\frac{1}{2} n,
\end{aligned}
$$

so we must have $|S| \leqq 11$.

$$
n \geqq|S|+5(|S|+2)
$$

Thus in any case, $|S| \leqq 11$. It remains finally to consider each possible value of $|S|$ in turn. In each case we argue as follows.

Let $h$ denote the number of vertices in all but one of the odd components of $G-S$. Then

whence

$$
|S|+h \geqq \frac{3-4 d-3 f}{2-2 d} h
$$

$$
|S| \geqq \frac{1-2 d-3 f}{2-2 d} h .
$$

Thus

$$
|S| \geqq \frac{1-2 d-3 f}{2-2 d} \cdot 5 \cdot(|S|+1) .
$$

For any specific value of $|S|$, (18) gives a lower bound for $d$, and so for $d n$. For example, if $|S|=5$, (18) yields

and

$$
d \geqq \frac{2}{5}-\frac{9}{5} f
$$

$$
d n \geqq \frac{2}{5} n-\frac{9}{5} \theta
$$

where $\theta=f n$. Having obtained this bound for $d n$, we obtain a contradiction by estimating $n$ in two different ways. For we have

and also

$$
n>|S|+5(|S|+\theta+1)
$$

$$
n>(|S|+\theta+1)(d n-|S|+1) \text {. }
$$

With $|S|=5$, these give

and

$$
n>35+5 \theta
$$

i.e.

$$
n>(6+\theta)\left(\frac{2}{5} n-\frac{2}{5} \theta-4\right)
$$

$$
n<\frac{9 \theta^{2}+74 \theta+120}{2 \theta+7} \text {. }
$$

(19) and (20) contradict one another. The theorem is now proved. 


\section{REFERENCES}

(1) I. Anderson, Perfect matchings of a graph, J. Combinatorial Theory 10 (1971), 183-186.

(2) C. BERGE, The theory of graphs and its applications (Methuen, London, 1962).

(3) W. T. Tutre, The factorisation of linear graphs, J. London Math. Soc. 27 (1947), 107-111.

(4) D. R. Woodall, The melting point of a graph, and its Anderson number (to appear).

The Department of Mathematics

UNIVERSITY GARDENS

GLASGOW G12 8QW 